\title{
Are Patients With Diabetes Mellitus at Increased Risk of COVID-19 Infection?
}

\author{
Mehmet Sözen $^{1^{*}}$ (D) Fatma Çölkesen ${ }^{2^{*}}$ (D) Şevket Arslan ${ }^{3}$ (D) Fatih Çölkesen ${ }^{3}$ \\ Melia Karaköse ${ }^{4}$ (I) İbrahim Erayman ${ }^{5}$ (D) Soner Demirbaş ${ }^{6}$ (I) Turgut Teke $^{6}$ (D)
}

\footnotetext{
1 Kocaeli University Medical Faculty, Department of Endocrinology and Metabolism, Kocaeli, Turkey

2 Konya Training and Research Hospital, Department of Infectious Diseases and Clinical Microbiology, Konya, Turkey.

3 Necmettin Erbakan University Meram Medical Faculty, Department of Allergy and Immunology, Konya, Turkey.

4 Necmettin Erbakan University Meram Medical Faculty, Department of Endocrinology and Metabolism, Konya, Turkey.

5 Necmettin Erbakan University Meram Medical Faculty, Department of Infectious Diseases and Clinical Microbiology, Konya, Turkey.

6 Necmettin Erbakan University Meram Medical Faculty, Department of Pulmonology, Konya, Turkey.
}

\begin{abstract}
Background: The aim of this study was to examine the clinical course and outcomes of patients with diabetes mellitus (DM) with coronavirus disease-2019 (COVID-19).

Methods: This retrospective, single-center study included 185 adult patients diagnosed with COVID-19. All patients were separated into 4 groups. Group 1(n=79): patients with no accompanying disease, Group 2 ( $n=14)$ : patients with only DM, Group 3 ( $n=31)$ : patients with comorbid disease(s) including DM, Group 4 (n=61): patients with comorbid disease(s) without DM. Data about COVID-19 management and outcome were obtained from the medical records of the patients. COVID-19 was confirmed by real-time polymerase chain reaction (RT-PCR) from throat swab samples. All patients underwent chest x-rays or chest computed tomography.

Results: 185 patients diagnosed with COVID-19 were evaluated. The COVID-19 prognoses of the patients were classified as good, moderate and poor. No statistically significant difference was determined between the groups in terms of COVID-19 prognosis ( $\mathrm{p}>0.05$ ). While the rate of DM patients with a good prognosis was $20.4 \%$, the DM patient rate increased up to $40 \%$ among moderate or poor prognosis patients. A statistically significant difference was observed between blood glucose levels and mortality (p: 0.008). Mortality due to COVID-19 pneumonia developed in $15(8.1 \%)$ patients. Mortality increase was mostly encountered in the group with DM and accompanying comorbidities. It was observed that ACEI / ARB use had no effect on mortality.

Conclusions: Although the study results do not show a statistically significant effect of DM on the prognosis of COVID-19 patients, the higher rate of DM patients in the group with poor prognosis suggests that it may affect the severity of COVID-19. These results may be useful for clinicians in the management of DM patients with COVID-19.
\end{abstract}

Key words: Diabetes Mellitus, Coronavirus, COVID-19, Prognosis, Mortality.

*Mehmet Sözen and Fatma Çölkesen contributed equally to this work

Cite this article as: Sözen M, Çölkesen F, Arsla Ş, Çölkesen F, Karaköse M, Erayman I, Demirbaş S, Teke T. Are Patients With Diabetes Mellitus at Increased Risk of COVID-19 Infection?. Arch Curr Med Res 2021;2(1):38-44 


\section{INTRODUCTION}

Coronaviruses $(\mathrm{CoV})$ are single-stranded, enveloped ribonucleic acid viruses that cause respiratory infections in humans (1). The coronavirus disease-2019 (COVID-19) caused by the severe acute respiratory syndromecoronavirus-2 (SARS-CoV-2) virus spread rapidly worldwide and was declared a pandemic by the World Health Organization (WHO) on March 11, 2020 (2).

Diabetes mellitus (DM) is becoming an increasing health problem worldwide and is recognized as one of the major risk factors for various infections and serious infectionrelated deaths. DM worsens the prognosis of infectious diseases, and these patients show increased morbidity and mortality rates for infection-related sepsis compared to the general population(3). Moreover, DM has recently been reported to be an important risk factor for mortality in patients infected with influenza A (H1N1) and coronavirus-associated Middle East respiratory syndrome (MERS-CoV) $(4,5)$.

Evidence from epidemiological studies in areas severely affected by SARS-CoV-2 and data from the Centers for Disease Control and Prevention (CDC) and other national health centers and hospitals suggest that death rates from COVID-19 are up to 50\% higher in individuals with DM than those who do not have DM (6). There are several hypotheses that explain the higher incidence of COVID-19 infection in people with DM. In general, people with DM are at an increased risk of infection due to innate phagocytosis, neutrophil chemotaxis, and defects affecting cell-mediated immunity. However, the high prevalence of DM in severe cases of COVID-19 could probably be associated with higher prevalence of DM in the elderly. Moreover, advanced age DM is associated with cardiovascular disease, which may also help explain the association with the fatal consequences of COVID-19 (7).

In this context, it has been suggested by many health authorities that DM should be in the group of high-risk diseases for COVID-19. However, there is still insufficient reliable data on DM characteristics in patients hospitalized due to COVID-19 (8).In this single-center retrospective study of 185 confirmed COVID-19 cases, the relationship between DM and clinical outcomes and mortality in COVID-19 patients were evaluated.

\section{MATERIALS AND METHODS}

Ethical Approval: The study was approved by the Local Ethics Committee of Necmettin Erbakan University, Meram Faculty of Medicine (Date: 03.07.2020, No: $2020 / 2664)$ and the study was conducted according to the Declaration of Helsinki, 1975.

This retrospective, single-center study included patients who presented at Necmettin Erbakan University, Meram Faculty of Medicine Hospital, Konya, Turkey, between March 15, 2020 and July 15,2020, and were diagnosed with COVID-19 according to the WHO interim guidance. According to the pandemic measures implemented by the Turkish Government, adult patients were admitted centrally to the hospital from the whole province of Konya without selectivity. All patients included in the study were hospitalized and treated.All the data regarding the patients in this study have been shared with the Ministry of Health. The epidemiological, demographic, clinical, laboratory, management, and outcome datawere obtained from the patient medical records. If data were missing from the records or clarification was needed, the attending doctors and other healthcare providers were contacted directly.

Nasal and throatswab specimens from the upper respiratory tract were obtained from all patients on admission and maintained in viral-transport medium. COVID-19 was confirmed with real-time polymerase chain reaction (RT-PCR). Sputum or endotracheal aspirates were obtained on admission for the identification of possible causative bacteria or fungi. All patients were applied with chest $\mathrm{x}$-rays or chest computed tomography (CT). According to the United States National Institutes of Health COVID-19 Treatment Guidelines, the prognosis of COVID-19 disease is divided into three groups as mild, moderate, or severe (9).

\section{Statistical analysis}

Statistical evaluations were made using IBM SPSS 20.0 software (IBM Corp., Armonk, NY, USA). Conformity of the data to normal distribution was evaluated with the Shapiro Wilk Test. Normally distributed numerical variables were stated as mean \pm standard deviation, non-normally distributed numerical variables as median (25th-75th percentile) values, and categorical variables as frequency (\%). Differences between groups were 
determined using t-test for numerical variables with normal distribution, Mann Whitney $U$ test for numerical variables without normal distribution, Kruskal Wallis test, One-Way Variance analysis and Dunn's multiple comparison test, and Fisher chi-square test for categorical variables, together with Yates chi-square, Pearson Chisquare and the Monte Carlo chi-square test. Relationships between variables were determined using Spearman Correlation Analysis. For testing two-sided hypotheses, a value of $\mathrm{p}<0.05$ was considered statistically significant.

\section{RESULTS}

185 patients with COVID-19 were evaluated. The patients were divided into 4 groups; Group $1(n=79)$ : patients with no accompanying disease, Group $2(n=14)$ : patients with only DM, Group $3(\mathrm{n}=31)$ : patients with comorbid disease (s) including DM; Group 4 ( $\mathrm{n}=61)$ : patinets with comorbid disease(s) without DM. None of the patients were medical staff. The chronic diseases of patients in Group 4 included cardiovascular, cerebrovascular, endocrine system, digestive system, respiratory system and nervous system diseases, and malignant tumours (Table 1).

Table 1: Numerical data of the patients enrolled in the study.

\begin{tabular}{|l|c|c|c|c|c|c|c|}
\hline \multicolumn{2}{|c|}{} & & Group 1 & Group 2 & Group 3 & Group 4 & Total \\
\hline \multirow{3}{*}{ Gender } & \multirow{3}{*}{ Female } & Count & 36 & 5 & 20 & 36 & 97 \\
\cline { 3 - 8 } & & $\%$ & $45.6 \%$ & $35.7 \%$ & $64.5 \%$ & $59.7 \%$ & $52.7 \%$ \\
\cline { 3 - 8 } & \multirow{3}{*}{ Male } & Count & 43 & 9 & 11 & 25 & 88 \\
\cline { 3 - 8 } & & $\%$ & $54.4 \%$ & $64.3 \%$ & $35.5 \%$ & $40.3 \%$ & $47.3 \%$ \\
\hline \multirow{3}{*}{ Total } & Count & 79 & 14 & 31 & 61 & 185 \\
\cline { 3 - 9 } & & $\%$ & $100.0 \%$ & $100.0 \%$ & $100.0 \%$ & $100.0 \%$ & $100.0 \%$ \\
\hline
\end{tabular}

The median (25th-75th percentile) age of the groups was; Group 1: 57 years (39.00-66.00),Group 2: 54.50 years (49.00-66.75),Group 3: 65 years (59-75), Group 4: 62.50 years (54.75-59.00). A statistically significant difference was observed between Group 1 and Group 3 considering median age $(\mathrm{p}<0.001)$ and there was no difference between the other groups.
When the HbA1c and fasting plasma glucose (FPG) levels of the patients with DM (Groups 2 and 3) were compared with each other, no statistically significant difference was found between the two groups $(p>0.05)$. All patients' DM was similarly regulated. All patients included in the study were hospitalized and followed up. The COVID-19 prognosis of the patients was classified as mild, moderate, or severe. There was no statistical difference between the four groups in terms of COVID-19 prognosis (Table 2).

Table 2: COVID-19 prognosis of the patients.

\begin{tabular}{|c|c|c|c|c|c|c|c|}
\hline \multicolumn{2}{|c|}{} & Group 1 & Group 2 & Group 3 & Group 4 & Total & pvalue \\
\hline \multirow{3}{*}{ Prognosis } & Mild & $63(79.7 \%)$ & $11(78.6 \%)$ & $18(58.1 \%)$ & $50(82.0 \%)$ & $142(76.8 \%)$ & $>0.05$ \\
\cline { 3 - 8 } & \multirow{2}{*}{\begin{tabular}{c} 
Moderate \\
\cline { 3 - 8 }
\end{tabular}} & $4(5.1 \%)$ & $1(7.1 \%)$ & $1(3.2 \%)$ & $2(3.3 \%)$ & $8(4.3 \%)$ & $>0.05$ \\
\cline { 3 - 8 } & Severe & $12(15.2 \%)$ & $2(14.3 \%)$ & $12(38.7 \%)$ & $9(14.8 \%)$ & $35(18.9 \%)$ & $>0.05$ \\
\hline
\end{tabular}

When all the DM patients (Groups 2 and 3) were compared with other COVID-19 positive patients (Groups 1 and 4) in terms of prognosis, there was no statistically significant difference between the groups (p:0.064). In the subgroup analyses, the majority of patients with a good prognosis $(79.6 \%)$ were non-DM patients. While the rate of DM patients with a good prognosis was $20.4 \%$, the DM patient rate increased up to $40 \%$ among moderate or poor prognosis patients (Table 3 ). 
Table 3: COVID-19 prognosis of the DM and non-DM patients.

\begin{tabular}{|l|c|c|c|c|c|}
\hline \multicolumn{2}{|c|}{} & Group 2+3 & Group 1+4 & Total & p value \\
\hline \multirow{3}{*}{ Prognosis } & Mild & $29(20.4 \%)$ & $113(79.6 \%)$ & $142(100.0 \%)$ & $>0.05$ \\
\cline { 3 - 6 } & Moderate & $2(25.0 \%)$ & $6(75.0 \%)$ & $8(100.0 \%)$ & $>0.05$ \\
\cline { 3 - 6 } & Severe & $14(40.0 \%)$ & $21(60.0 \%)$ & $35(100.0 \%)$ & $>0.05$ \\
\hline \multicolumn{2}{|l|}{ Total } & $45(24.3 \%)$ & $140(75.7 \%)$ & $185(100.0 \%)$ & $>0.05$ \\
\hline
\end{tabular}

Of all the patients included in the study, 167 were discharged from the hospital. Of these, 75 had a negative COVID-19 PCR result at the time of discharge, and the remaining patients were discharged with instructions to continue isolation at home. Hospitalization of 3 patients continued, and mortality developed in $15(8.1 \%)$ due to
COVID-19 pneumonia. Only 3 of the patients who died had no history of chronic disease. The remaining 12 patients had at least one chronic disease. Mortality was mostly observed in the group including patients with DM and comorbid diseases (Group 3). Mortality did not occur in any of the 14 patients (Group 2) with only DM (Table 4).

Table 4: The condition of the patients enrolled in the study after treatment.

\begin{tabular}{|l|c|c|c|c|c|}
\hline & Group 1 & Group 2 & Group 3 & Group 4 & Total \\
\hline \multirow{2}{*}{$\begin{array}{l}\text { Inhospital } \\
\text { Discharged }\end{array}$} & $1(1.3 \%)$ & $1(7.1 \%)$ & $1(3.2 \%)$ & $0(0.0 \%)$ & $3(1.6 \%)$ \\
\cline { 2 - 6 } $\begin{array}{l}\text { PCR (-) } \\
\text { discharge }\end{array}$ & $38(48.1 \%)$ & $8(57.1 \%)$ & $14(45.2 \%)$ & $32(53.2 \%)$ & $92(50.0 \%)$ \\
\cline { 2 - 6 } Exitus & $37(46.8 \%)$ & $5(35.7 \%)$ & $8(25.8 \%)$ & $25(40.3 \%)$ & $75(40.3 \%)$ \\
\hline Total & $79(100.0 \%)$ & $14(100.0 \%)$ & $31(100.0 \%)$ & $4(6.5 \%)$ & $15(8.1 \%)$ \\
\hline
\end{tabular}

Considering the final status of the patients, a statistically significant difference was observed between blood glucose levels and mortality. The median blood glucose level of the patients who died was statistically significantly higher than that of patients who were discharged (p:0.008).
When the effect of angiotensin converting enzyme inhibitors (ACEI) and angiotensin II receptor blockers (ARB) on COVID-19 prognosis was evaluated, no significant negative effectwas observed ( $p>0.05$ ) (Table 5). No significant difference was observed between surviving and exitus patients consideringthe ACEI / ARB usage rate (p:0.102) (Table 6).

Table 5: The effect of drug use on disease prognosis.

\begin{tabular}{|l|c|c|c|c|c|}
\hline & Mild & Moderate & Severe & Total & p value \\
\hline \multirow{2}{*}{$\begin{array}{l}\text { ACEI / ARB users } \\
\text { Drug free }\end{array}$} & $31(77.5 \%)$ & $1(2.5 \%)$ & $8(20.0 \%)$ & $40(100.0 \%)$ & $>0.05$ \\
\cline { 2 - 5 } Other drugs & $68(78.2 \%)$ & $5(5.7 \%)$ & $14(16.1 \%)$ & $87(100.0 \%)$ & $>0.05$ \\
\cline { 2 - 6 } & $43(74.1 \%)$ & $2(3.4 \%)$ & $13(22.4 \%)$ & $58(100.0 \%)$ & $>0.05$ \\
\hline Total & $142(76.7 \%)$ & $8(4.3 \%)$ & $35(18.9 \%)$ & $185(100.0 \%)$ & $>0.05$ \\
\hline
\end{tabular}


Table 6: The effect of drug use on mortality.

\begin{tabular}{|l|c|c|c|c|}
\hline & Exitus & Surviving & Total & p value \\
\hline \multirow{2}{*}{ ACEI / ARB users } & $6(15 \%)$ & $34(75 \%)$ & $40(100.0 \%)$ & $>0.05$ \\
\cline { 2 - 5 } Other drugs & $9(6.2 \%)$ & $136(93.8 \%)$ & $145(100.0 \%)$ & $>0.05$ \\
\hline Total & $15(8.1 \%)$ & $170(91.9 \%)$ & $185(100.0 \%)$ & $>0.05$ \\
\hline
\end{tabular}

\section{DISCUSSION}

Diabetes mellitus (DM) is one of the leading causes of mortality and morbidity worldwide and is expected to increase significantly in the coming years. DM is associated with a variety of vascular, cardiac, and renal complications that affect the overall survival of patients, and patients with DM are generally more susceptible to infections (10). Infectious diseases such as influenza and pneumonia are very common among elderly diabetic patients.In previous studies, DM has been shown to be a risk factor for the mortality and morbidity of many viral infections, including H1N1, MERS-CoV and SARS-CoV $(10,11)$.

In this retrospective cohort study, data from 185 patients with COVID-19 were analyzed, including 45 patients with DM and 140 patients without DM. Age distribution of the patients was compared among the four groups. It was observed that patients with comorbid diseases were older than others. Patients with DM and comorbidity in particular constituted the oldest population. It is well known that the incidence of DM increases with aging, and some studies have shown that old age is one of the most important risk factors affecting the prognosis of COVID-19 (12). Therefore, a poor clinical outcome may be predicted for a large proportion of diabetic elderly patients.

In a study evaluating the severity of COVID-19 in DM patients, Wu et al found a higher rate of diabetes in severe cases of COVID-19 compared to mild cases (13). Similarly, in another study, COVID-19 patients with DM were more likely to develop severe or critical illnesses. In addition, these patients had a greater need for invasive or noninvasive mechanical ventilation and higher mortality rates (14). In a study by Liu et al., the percentage of patients with diabetes among the severe and critical COVID-19 cases among 1880 COVID-19 patients was higher than mild or moderate cases. However, there was no difference between the patients with and without DM during the follow-up period. Similar mortality rates were found in patients with or without DM $(2.9 \%(\mathrm{n}=4)$ and $1.1 \%$ (n =9), respectively (p:0.114)) (15). In the current study, no statistically significant difference was determined between the four groups in terms of COVID-19 prognosis, which is consistent with some data in the literature. However, when all DM patients were compared to those without $\mathrm{DM}$, worse prognoses were mostly encountered in DM patients. This may partly explain the higher proportion of severe or critical COVID-19 cases in the groups with DM than in the groups without DM.

Recent studies have revealed that DM is one of the most common comorbidities in COVID-19 infection (16). Furthermore, some studies have suggested that COVID-19 patients with DM have a higher risk of death during infection $(16,17)$. In a study of 1382 patients by Roncon et al, higher rates of intensive care unit admission and a higher risk of mortality were reported in DM COVID-19 patients (18). In another study reporting 1880 COVID-19 patients, the mortality rate of patients with and without DM was $2.9 \%(\mathrm{n}=4)$ and $1.1 \%(\mathrm{n}=9)$, respectively (p:0.114) (15). The mortality rates in the currentstudy were found to increase with accompanying comorbid diseases. The highest mortality rate was observed in the patient group with comorbid disease(s) including DM. These data support many studies in the literature reporting increase in severity and mortality rates for COVID-19 patients who have DM.

ACEI and ARB drugs are recommended by the National Institute for Health and Care Excellence as first-line treatment for patients under 55 years of age with hypertension, as second-line treatment for those over 55 years of age and for those of African descent (19). However, the use of ACEI and ARB in COVID-19 patients has caused great debate because ACE-2 is a surface receptor 
for SARS-CoV-2 (20). Previous research has shown that SARS-CoV-2 binds to ACE-2 enzyme at low cytosolic $\mathrm{pH}$ values, entering the cell and causing infection (21). Angiotensin II has a strong $\mathrm{pH}$ alkaline effect, which causes alkalinizationin the environment $\mathrm{pH}$ even after strong acid loading (22). ACEIs and ARBs lead to a reduction in angiotensin II level by increasing the ACE2 level, thereby causing a low cytosolic Ph (21). The presence of ACE2 in many organs such as the cardiovascular system, lungs, kidneys and brain may explain why some COVID-19 patients die from multiple organ failure (23). It has been suggested that the mortality and morbidity rates would be higher in COVID-19 patients with DM if they were using ACEI and ARB (21).

In a cohort study including 19486 patients with COVID-19 disease in the United Kingdom, ACEI were associated with a significantly reduced risk of COVID-19 disease. In the same study, no increase was found in the risk of intensive care unit hospitalization. There were significant interactions between ethnicity and ACEI and ARB use for risk of COVID-19 disease. The risk of COVID-19 disease associated with ACEI use was higher in Caribbean and Black African groups than the white group. A higher risk of COVID-19 associated with ARB was observed for Black African group compared to the white group (24). In a multicenter retrospective study of 1128 adult patients with hypertension diagnosed with COVID-19, the results consistently demonstrated a lower risk of COVID-19 mortality in patients who received ACEI/ARB than those who did not receive ACEI/ARB (25). Conversely, in another study there was no evidence of a reduced risk of results in patients taking ACEI and ARB medications (26). In the current study, the prescription of ACEI and ARB drugs was not determined to have a significant effect on mortality. However, the prognosis for COVID-19 was significantly better in ACEI/ ARB users.

A few limitations of this study should be addressed. First, the retrospective, non-randomized design led to sample heterogeneity. Second, although maintaining blood glucose regulation can affect the clinical course and outcome of COVID-19, there was insufficient analysis of thedata on anti-DM treatment. Third, the relative mechanism behind the impact of diabetes on COVID-19 was not examined in this study. Fourth, the clinical outcome data includes data of patients within a specified period after hospitalization.
The absence of data of the hospitalized patients at the end of the pre-defined study period may have affected the findings, including mortality rates. Finally, the difference in disease progression and prognosis between COVID-19 patients with or without DM may change with a longer follow-up period.

In conclusion, whether DM significantly affects the prognosis of COVID-19 is still a matter of debate. The results of this study showed that DM had no significant effect on the prognosis of COVID-19, but the increased rate of DM patients in the poor prognosis group suggests that it may be related to the severity of the disease. This study may assist clinicians in managing COVID-19 patients with DM. However, prospective studies with larger patient populations will more clearly reveal the impact of DM on COVID-19 prognosis.

\section{Declarations}

The authors received no financial support for the research and/or authorship of this article. There is no conflict of interest.

Ethical Approval: The study was approved by the Local Ethics Committee of Necmettin Erbakan University, Meram Faculty of Medicine (Date: 03.07.2020, No: 2020/2664) and the study was conducted according to the Declaration of Helsinki, 1975.

\section{REFERENCES}

1. Cui J, Li F, Shi ZL. Origin and evolution of pathogenic coronaviruses. Vol. 17, Nature Reviews Microbiology. Nature Publishing Group; 2019. p. 181-92.

2. Huang C, Wang Y, Li X, Ren L, Zhao J, Hu Y, et al. Clinical features of patients infected with 2019 novel coronavirus in Wuhan, China. Lancet. 2020 15;395(10223):497-506.

3. Frydrych LM, Bian G, O'Lone DE, Ward PA, Delano MJ. Obesity and type 2 diabetes mellitus drive immune dysfunction, infection development, and sepsis mortality. J Leukoc Biol. 2018;104(3):525-34.

4. Alqahtani FY, Aleanizy FS, Ali El Hadi Mohamed R, Alanazi MS, Mohamed N, Alrasheed MM, et al. Prevalence of comorbidities in cases of Middle East respiratory syndrome coronavirus: A retrospective study. 2018;147:1-5

5. Yang JK, Feng Y, Yuan MY, Yuan SY, Fu HJ, Wu BY, et al. Plasma glucose levels and diabetes are independent predictors for mortality and morbidity in patients with SARS. Diabet Med. 2006;23(6):623-8.

6. Remuzzi A, Remuzzi G. COVID-19 and Italy: what next? Vol. 395, The Lancet. Lancet Publishing Group; 2020. p. 1225-8.

7. Bornstein SR, Rubino F, Khunti K, Mingrone G, Hopkins D, Birkenfeld $\mathrm{AL}$, et al. Practical recommendations for the management of diabetes in patients with COVID-19. Vol. 8, The Lancet Diabetes and Endocrinology. Lancet Publishing Group; 2020. p. 546-50. 
8. CDC. Certain Medical Conditions and Risk for Severe COVID-19 Illness I CDC. Avaliable at: https: / /www.cdc.gov/ coronavirus / 2019ncov/need-extra-precautions / people-with-medical-conditions.html. Accessed November 29, 2020.

9. CDC. Certain Medical Conditions and Risk for Severe COVID-19 Illness | CDC. Avaliable at: https://www.cdc.gov/ coronavirus/2019ncov/need-extra-precautions/people-with-medical-conditions.html. Accessed November 29, 2020.

10. NIH. What's new | COVID-19 Treatment Guidelines. 2020. Available at: https://www.covid19treatmentguidelines.nih.gov/whats-new/. Accessed November 29, 2020.

11. Knapp S. Diabetes and Infection: Is There a Link? - A Mini-Review. Gerontology. 2013;59(2):99-104.

12. Banik GR, Alqahtani AS, Booy R, Rashid H. Risk factors for severity and mortality in patients with MERS-CoV: Analysis of publicly available data from Saudi Arabia. Vol. 31, Virologica Sinica. Science Press; 2016. p. 81-4.

13. Zhou F, Yu T, Du R, Fan G, Liu Y, Liu Z, et al. Clinical course and risk factors for mortality of adult inpatients with COVID-19 in Wuhan, China: a retrospective cohort study. Lancet. 2020;395(10229):1054-62.

14. Wu J, Li W, Shi X, Chen Z, Jiang B, Liu J, et al. Early antiviral treatment contributes to alleviate the severity and improve the prognosis of patients with novel coronavirus disease (COVID-19). J Intern Med. 2020;288(1):128-38.

15. Zhang Y, Cui Y, Shen M, Zhang J, Liu B, Dai M, et al. Association of diabetes mellitus with disease severity and prognosis in COVID-19: A retrospective cohort study. Diabetes Res Clin Pract. 2020;165:108227.

16. Liu Z, Li J, Huang J, Guo L, Gao R, Luo K, et al. Association Between Diabetes and COVID-19: A Retrospective Observational Study With a Large Sample of 1,880 Cases in Leishenshan Hospital, Wuhan. Front Endocrinol (Lausanne). 2020;11:478.

17. Gupta R, Ghosh A, Singh AK, Misra A. Clinical considerations for patients with diabetes in times of COVID-19 epidemic. Vol. 14, Diabetes and Metabolic Syndrome: Clinical Research and Reviews. Elsevier Ltd; 2020. p. 211-2.

18. Ruan Q, Yang K, Wang W, Jiang L, Song J. Clinical predictors of mortality due to COVID-19 based on an analysis of data of 150 patients from Wuhan, China. Vol. 46, Intensive Care Medicine. Springer; 2020. p. 846-8.

19. Roncon L, Zuin M, Rigatelli G, Zuliani G. Diabetic patients with COVID-19 infection are at higher risk of ICU admission and poor short-term outcome. J Clin Virol. 2020;127:104354.

20. National Institute for Health and Care Excellence Hypertension in adults: diagnosis and management. NICE guideline, 2019. Available at: https: / / www.nice.org.uk/guidance/ng136/resources/hypertensionin-adults-diagnosis-and-management-pdf-66141722710213. Accessed November 23, 2020
21. Kuba K, Imai Y, Rao S, Gao H, Guo F, Guan B, et al. A crucial role of angiotensin converting enzyme 2 (ACE2) in SARS coronavirusinduced lung injury. Nat Me. 2005;11(8):875-9.

22. Cure E, Cumhur Cure M. Comment on "Organ-protective effect of angiotensin-converting enzyme 2 and its effect on the prognosis of COVID-19." J Med Virol. 2020;92(9):1423-4.

23. Da Costa-Pessoa JM, Dos Santos Ruiz Figueiredo CF, Thieme K, Oliveira-Souza M. The regulation of NHE1 and NHE3 activity by angiotensin II is mediated by the activation of the angiotensin II type i receptor/phospholipase $\mathrm{C} /$ calcium/calmodulin pathway in distal nephron cells. Eur J Pharmacol. 2013;721(1-3):322-31.

24. Li MY, Li L, Zhang Y, Wang XS. Expression of the SARS-CoV-2 cell receptor gene ACE2 in a wide variety of human tissues. Infect Dis Poverty. 2020;9(1):45.

25. Hippisley-Cox J, Young D, Coupland C, Channon KM, Tan PS, Harrison DA, et al. Risk of severe COVID-19 disease with ACE inhibitors and angiotensin receptor blockers: cohort study including 8.3 million people. Heart. 2020;106(19):1503-11.

26. Zhang P, Zhu L, Cai J, Lei F, Qin JJ, Xie J, et al. Association of Inpatient Use of Angiotensin-Converting Enzyme Inhibitors and Angiotensin II Receptor Blockers with Mortality among Patients with Hypertension Hospitalized with COVID-19. Circ Res. 2020;126(12):1671-81.

27. Richardson S, Hirsch JS, Narasimhan M, Crawford JM, McGinn T, Davidson KW, et al. Presenting Characteristics, Comorbidities, and Outcomes Among 5700 Patients Hospitalized With COVID-19 in the New York City Area. Jama. 2020;323(20):2052. 